\title{
Heterogene Therapieziele für eine heterogene Population
}

\author{
Individualisierung lautet das Zauberwort in der Typ- \\ 2-Diabetestherapie. Patienten reagieren sehr unter- \\ schiedlich auf die antihyperglykämische Therapie. \\ Prädiktoren helfen abzuschätzen, wer besonders pro- \\ fitieren wird und wem Risiken drohen.
}

Vor ziemlich genau 17 Jahren begann die Begeisterung für die strikt normnahe Blutzuckereinstellung bei allen Typ-2-Diabetikern, erinnerte Prof. Dr. Stefano Del Prato, Universität Pisa. Die United Kingdom Prospective Diabetes Study (UKPDS) hatte schließlich ergeben, dass praktisch alle klinischen Endpunkte durch eine gute Stoffwechselkontrolle reduziert wurden.

Beim Myokardinfarkt war das Ergebnis zwar zunächst nicht signifikant, wurde es aber im Langzeitverlauf. Das galt als Beweis für das metabolische Gedächtnis des Organismus und wurde zum Anlass genommen, den $\mathrm{HbA}_{1 c}$-Zielwert einheitlich auf $<6,5-7,0 \%$ festzuschreiben.

Dass spätere Studien den Erfolg nicht wiederholen konnten, teilweise sogar negative Effekte einer aggressiven Blutzuckersenkung zeigten, wurde vor allem auf den verzögerten Therapiebeginn - direkt nach Diagnose in UKPDS, zehn Jahre später z. B. in VADT - und auf die hohe Hypoglykämie-Inzidenz zurückgeführt.

Dass Hypoglykämien das kardiovaskuläre Risiko erhöhen, ist aus pathophysiologischer Sicht mehr als plausibel. Zum einen verstärken sie die Koagulabilität, weil Plättchenzahl und Aggregationsneigung ebenso zunehmen wie die Synthese bestimmter Gerinnungsfaktoren (Faktor VIII, von Willebrand-Faktor).

Zum anderen steigt die Ausschüttung proinflammatorischer Zytokine, die Endothelfunktion wird beeinträchtigt mit der Folge einer gesteigerten Vasokonstriktion. „Die in VADT eingetretenen kardiovaskulären Komplikationen sind aber mindestens so sehr Folge der Schäden, welche die Hyperglykämie vorher bereits hinterlassen hat, wie der strengen Blutzuckereinstellung", meinte Del Prato.

\section{Blutzuckersenkung als ein Baustein}

Auch bei VADT zeigte sich übrigens im Langzeitverlauf ein Rückgang der Myokardinfarkte, der noch dazu in gleicher Größenordnung lag wie bei UKPDS, nämlich bei etwa 15\%. „Im Vergleich zu anderen Interventionen wie der Blutdruck- oder Lipidsenkung, aber auch im Vergleich zu anderen diabetischen Endpunkten ist das nicht viel, aber es ist auch nicht nichts", betonte Del Prato. Er plädiert für den ganzheitlichen Ansatz: „Zu denken, Glukosesenkung lohnt sich nicht, ich gebe mein Geld lieber für etwas anderes aus, vereinfacht und greift zu kurz.“

Der italienische Kardiologe erinnerte außerdem daran, dass Typ-2-Diabetiker eine heterogene Population sind, die eine individualisierte Therapie geradezu erzwingen. Das Vorliegen einer chronischen Nierenschädigung beispielsweise erschwert es nicht nur, angestrebte $\mathrm{HbA}_{1 c}$-Ziele zu erreichen. Es verschlechtert auch die Chancen auf eine herz- und gefäßprotektive Wirkung der antiglykämischen Therapie über alle Endpunkte von Schlaganfall und Herzinfarkt bis hin zum Tod. Es steigert au-

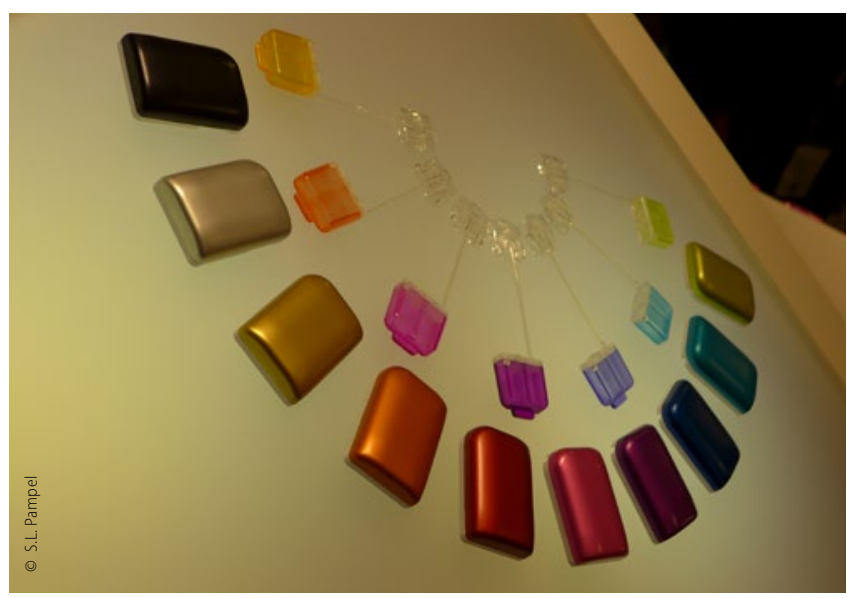

So heterogen wie die Therapieziele ist die Farbpalette der Insulinpumpen eines neuen niederländschen Herstellers.

Berdem das Risiko, dass der Patient unter intensiver Blutzuckersenkung in eine schwere Hypoglykämie rutscht.

\section{Glykierungs-Index identifiziert Patienten, die profitieren}

Möglicherweise könnte der Hemoglobin Glycation Index (HGI) künftig helfen, Patienten zu identifizieren, bei denen sich eine intensive antiglykämische Therapie besonders lohnt. Der Index basiert darauf, dass bei gegebenem Glukosespiegelverlauf nicht bei jedem Diabetiker gleich viel $\mathrm{HbA}_{1 c}$ entsteht. Dazu wird der tatsächlich gemessene $\mathrm{HbA}_{1 c}$-Wert in Relation zu dem anhand der Nüchternglukose ermittelten erwarteten Wert gesetzt.

Eine Nachauswertung der ACCORD-Studie - neben VADT und ADVANCE die dritte Endpunktstudie zur intensiven versus konventionellen antiglykämischen Therapie, die mit einer Enttäuschung geendet hatte - hat ergeben, dass Patienten mit hohem HGI nicht von der intensivierten Therapie profitierten.

Im Gegenteil: Ihr Hypoglykämie-, kardiovaskuläres Komplikations- und Sterberisiko stieg sogar deutlich an, während Patienten mit niedrigem oder mittlerem HGI ein signifikant verringertes kardiovaskuläres Risiko aufwiesen.

Für Del Prato steht damit fest, dass eine moderne antidiabetische Therapie sich nicht auf das Motto „One size fits all“ stützen darf. Vielmehr gilt, den Patienten in all seinen Facetten zu betrachten, mit seinem biologischen Alter und seiner Restlebenserwartung, seiner Diabetesdauer, seinen diabetischen Folge- und Begleiterkrankungen, um am Ende zu individuellen Therapiezielen zu gelangen.

Der Zielwert kann für frisch erkrankte, biologisch fitte Typ2-Diabetiker durchaus unter 6,5\% liegen. Bei älteren multimorbiden Patienten dürften Werte zwischen 7,5 und 8,5\% aber nicht nur realistischer, sondern auch prognostisch günstiger sein.

Manuela Arand

Quelle: EASD-Jahreskongress 2015 vom 14.-18.9.2015 in Stockholm 